\title{
Deducing Immortality
}

\author{
Aprajita* \\ Stem Cell Engineering, India
}

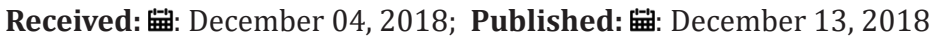

*Corresponding author: Aprajita, Stem Cell Engineering at Barkatullah University, Bhopal, India

\section{Abstract}

Stem cells can express telomerase while telomerase activity is widely suppressed in somatic cell, resulting in senescence because of telomere shortening during replication. The level of expression of telomerase expressing gene (hTERT gene) is lower in adult stem cell whereas high in embryonic and cancer stem cell. Till now the level of hTERT expression is not even studied in pluripotent stem cell, as c-Myc gene plays a major role in up regulating hTERT. This study is about the interconnection between c-Myc being a stem cell pluripotency factor and an up regulator for telomerase.

Keywords: Chromosome; Transcription; Clustal Omega; Reprogrammed Gene

Abbreviations: hTERT: Telomerase Reverse Transcriptase in Humans; RNA: Ribonucleic Acid; IPSC: Induced Pluripotent Stem Cells

\section{Introduction}

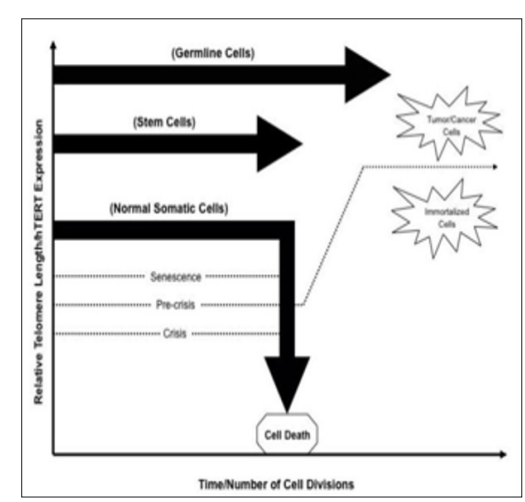

Figure 1: Diagram showing the telomere length in somatic cell, germ cell and stem cell and how any mutation in hTERT gene can lead to cancer cell formation.

Telomere defined as a region of repetitive sequence at each end of eukaryotic chromosome which is shortened as a result of incomplete replication of linear chromosome, the so called "end replication problem". The maintenance of telomerase has been proposed as an essential to human tumor development and stem cells are known to overcome telomerase shortening by expressing enzyme called Telomerase. Telomerase is a complex of reverse transcriptase protein which is expressed by hTERT gene and adds a species dependent telomere repeat sequence to 3'end of telomere [1]. Basically, the hTERT gene which is located on the short arm of human chromosome 5 (5p15.33) expresses Human Telomerase reverse transcriptase. Human Telomerase reverse transcriptase is a catalytic subunit of the enzyme telomerase, which, together with the telomerase RNA component (TERC), comprises the important unit of the enzyme telomerase complex. It is thought that the hTERT promoter is the most important regulatory element of telomerase expression. The G-rich sequences located within the hTERT promoter have a potential to form G-quadruplexes which are helical structures composed of four-strands and formed via the stacking of GGGG tetrads. This Quadruplex formation within the telomeric G-rich overhang has been shown to prevent its elongation by telomerase negatively impacting c-Myc expression and in turn the expression of hTERT (Figures $1 \& 2$ ).

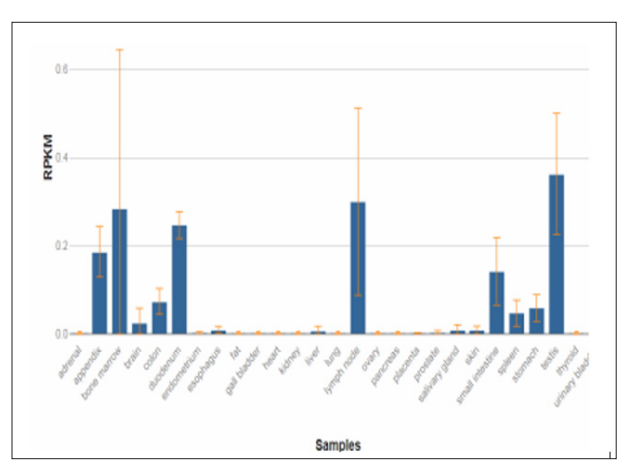

Figure 2: This data is collected from NCBI data bank, here hTERT expression is explained in terms of RPKM (i.e., Reads per kilo base of transcript, per million mapped reads) for different tissues in human body. 


\section{Activators of hTERT}

\section{Transcription Factors and Hormones}

The up regulator or activator of hTERT include several transcription factors like SP1, HER2, NFAT, which directly or indirectly activate via level of transcription. Sequential induction of nuclear factor kappa beta and Myc has been suggested to aid human epidermal growth factor receptor 2 in turn activating hTERT. P13K /AkT an MAP Kinase Pathways are known to up regulate hTERT whereas the TGF- beta pathway down regulate by suppressing Myc oncogene (Figure 3).

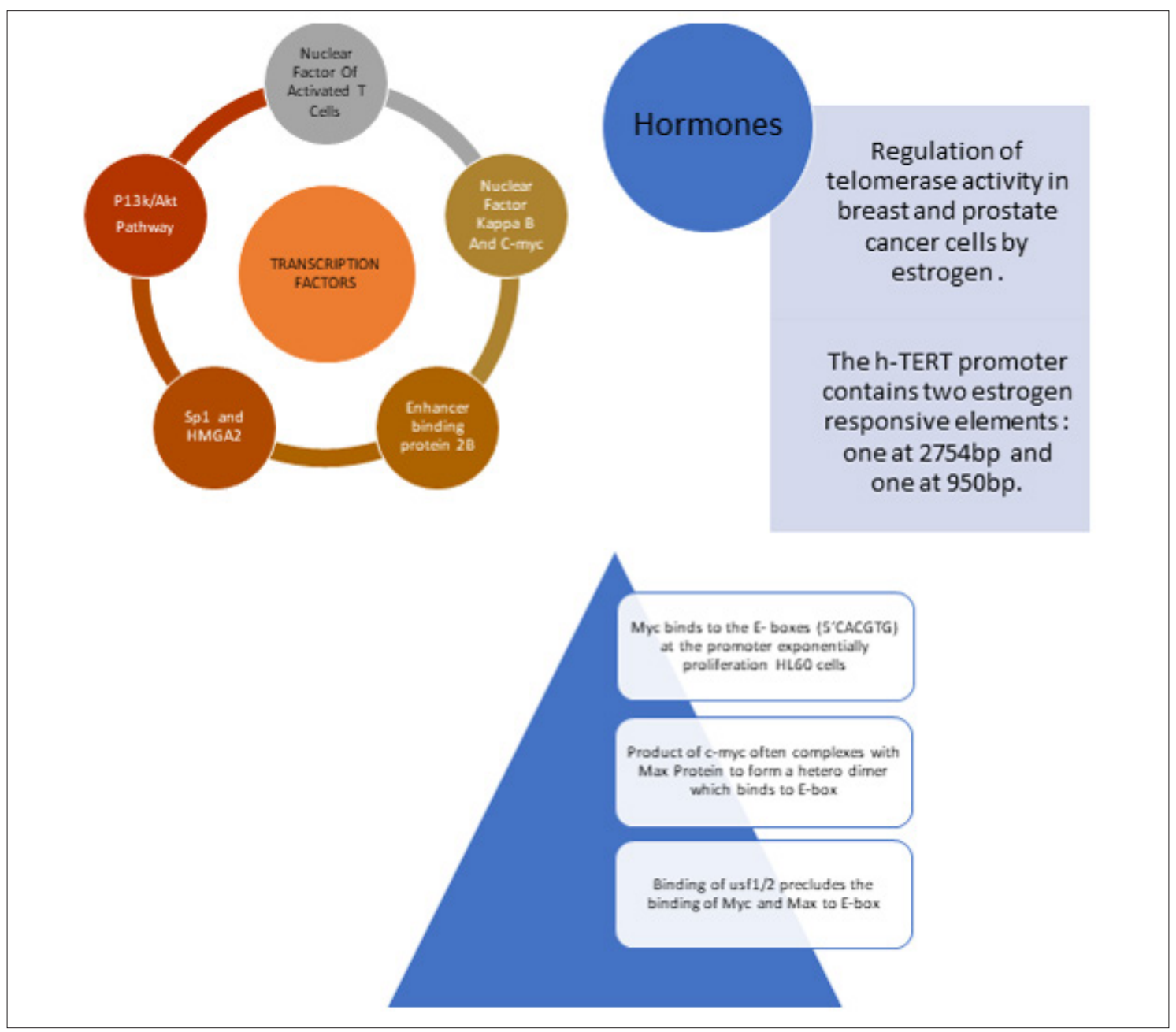

Figure 3: Diagram depicting all activators of hTERT gene and their importance in activation pathways.

\section{c-Myc: A Correlating Factor}

c-Myc as Gene Up Regulator Factor: c-Myc is an oncogene that is generally involved in management of cellular proliferation, differentiation and apoptosis in human somatic cells .c-Myc protein levels are known to elevated in undifferentiated and transformed cells. In an experiment by Jing Wang et al, the introduction of a c-Myc expression cassette stimulated telomerase activity in Human mammary epithelial cells. The enzyme activity was shown elevated within one passage after transduction of HMECS with a retro virus that directs Myc expression. The Myc -expressing populations displayed levels of telomerase activity that approximated those in breast carcinoma cell lines. c- Myc activates telomerase, an enzyme which is itself a marker for tumor cell and known to expressed by stem cells in order divide maximally.
Myc as a Reprogramming Factor: c-Myc also plays major role in the generation of induced pluripotent stem cells (iPSCs). It one of the original factors discovered by Yamanaka et al. to encourage cells to return to a 'stem-like' state alongside transcription factors Oct4, Sox 2 and Klf4). It has since been shown that it is possible to generate iPSC without c-Myc.

\section{Relating Stem Cells with Telomerase Dynamics}

\section{Cancer Stem Cells}

Telomerase expression in HSCs is considered as risk factor for development of chromosomal instability and malignant transformation in cancer stem cell. It is being thought that CSCs might have acquired immortality by mutational events in telomerelengthening mechanisms, typically the activation of telomerase. The 
inhibition of telomerase in CSCs to limit proliferation capacity and induce apoptosis and the identification of specific marker proteins in CSCs in each organ are likely to optimize therapeutic anticancer strategies.

\section{Future Possibilities and Conclusion}

There are several future possibilities of research on this interconnection of c-Myc like, it may be possible to find that the reprogrammed stem cells (iPSCs) using c-Myc, may have unregulated telomerase activity as compared to normal production. It could possible to target Myc for treating issues with cancer stem cells.

c-Myc is an oncogene which has a large scope for unfolding major genetic forefronts of stem cell and tumor science. Although, there is not a single factor contributing in senescence pathways but up regulation and down regulation of major transcription factors

\section{ISSN: 2574-1241}

DOI: $10.26717 / B J S T R .2018 .12 .002203$

Aprajita. Biomed J Sci \& Tech Res

(C) (P) This work is licensed under Creative Commons Attribution 4.0 License

Submission Link: https://biomedres.us/submit-manuscript.php like c-Myc, Sp1, Mdm-2 etc. plays a major role in inhibition and over expression of telomerase during tumorigenesis and stem cell proliferation. Myc is thus viewed as a promising target for anticancer drugs and as a recombinant factor [2-4].

\section{References}

1. EHiyama, K Hiyama (2007) Telomere and Telomerase In Stem Cells. British Journal of Cancer 96(7): 1020-1024.

2. Gunnar von Heijne, Anders Liljas (2005) Telomeres and Telomerase: Their Mechanisms of Action and the Effects of Altering their Function. $579(4)$.

3. Jing Wang, Lin Ying Xie, Susan Allan, David Beach, Gregory J Hannon (1998) Myc Activates Telomerase. Genes and Development 12(12): $1769-1774$.

4. Michael Daniel, Gregory W Peek, Trygve 0 Tollefsbol (2012) Regulation of The Human Catalytic Subunit of Telomerase (hTERT). Gene 498(2): 135-146.

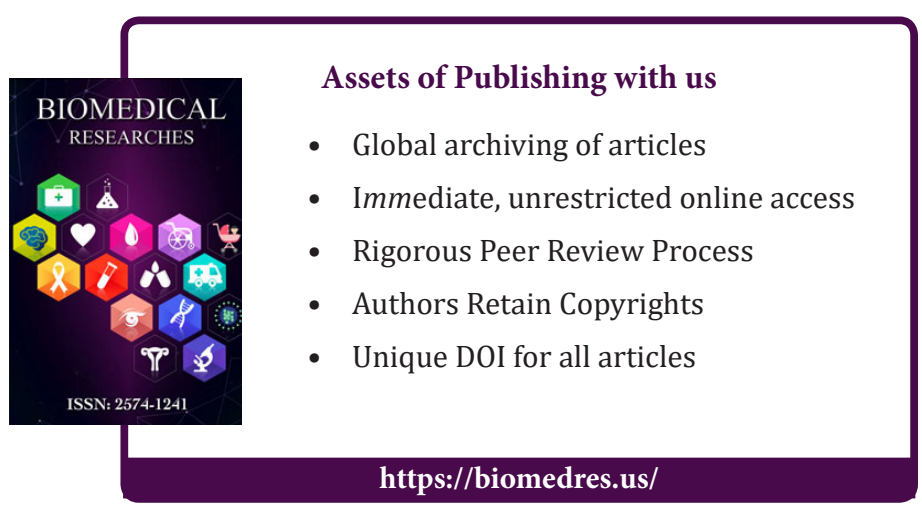

